\title{
Filmography and theatre credits
}

\section{As producer}

Every Day Except Christmas, 1957, 40 mins, b/w

Production company: Graphic Films, for the Ford Motor Company. First film of the series, 'Look at Britain'

Producers: Karel Reisz and Leon Clore

Director: Lindsay Anderson

Photography: Walter Lassally

Editing/Sound: John Fletcher

Music: Daniel Paris

Commentary: Alun Owen

I Want to Go to School, 1960, 30 mins, b/w

Production company: Graphic Films, for the National Union of Teachers

Producers: Karel Reisz and Leon Clore

Director and screenplay: John Krish

This Sporting Life, 1963, 134 mins, b/w

Production company: Independent Artists

Executive producers: Julian Wintle and Leslie Parkyn

Producer: Karel Reisz

Director: Lindsay Anderson

Screenplay: David Storey, adapted from his novel

Photography: Denys Coop

Art director: Alan Withy

Set decoration: Peter Lamont

Production director: Albert Fennell

Editing: Peter Taylor

Music/Musical director: Roberto Gerhard

Costumes: Sophie Devine

Casting: Miriam Brickman

Continuity: Pamela Mann 
Sound editing: Chris Greenham

Sound recording: John W. Mitchell and Gordon K. McCallum

Leading players: Richard Harris (Frank Machin), Rachel Roberts (Margaret Hammond), Alan Badel (Weaver), William Hartnell (Johnson), Colin Blakely (Maurice Braithwaite), Vanda Godsell (Mrs Weaver), Anne Cunningham (Judith), Jack Watson (Len Miller), Arthur Lowe (Slomer), Harry Markham (Wade), George Sewell (Jeff), Leonard Rossiter (Phillips), Katharine Parr (Mrs Farrer), Bernadette Benson (Lynda), Andrew Nolan (Ian), Peter Duguid (Doctor), Wallas Eaton (Waiter), Anthony Woodruff (Head waiter), Michael Logan (Riley), Murray Evans (Hooker), Tom Clegg (Gower), Ken Traill (Trainer), Frank Windsor (Dentist), John Gill (Cameron).

\section{As director}

Momma Don't Allow, 1956, 22 mins, b/w (co-directed with Tony Richardson)

Production company: British Institute, Experimental Film Production Fund

Co-director: Tony Richardson

Photography: Walter Lassally

Editing/Sound: John Fletcher

Music: The Chris Barber Band, with Chris Barber (trombone), Pat Halcox (trumpet), Monty Sunshine (clarinet), Lonnie Donegan (guitar), Jim Bray (counterbass), Ron Bowden (drums), Ottilie Patterson (vocals)

March to Aldermaston, 1959, 33 mins, b/w

Production company: Contemporary Films

Producer: Derrick Knight

Production assistants: Karel Reisz, Lindsay Anderson, Christopher Brunel, Charles Cooper, Allan Forbes, Derrick Knight, Kurt Lewenhak, Lewis McLeod, Eda Segal, Elizabeth Russell, Stephen Peet, Derek York.

Editing: Mary Beales and Lindsay Anderson

Commentary written by Christopher Logue and Lindsay Anderson, read by Richard Burton

We Are the Lambeth Boys, 1959, 52 mins, b/w

Production company: Graphic Films, for the Ford Motor Company. Second film of the series, 'Look at Britain'

Executive Producer: Robert Adams

Producer: Leon Clore

Assistants: Louis Wolfers, Raoul Sobel

Photography: Walter Lassally

Editing: John Fletcher 
Music: John Dankworth and his Orchestra

Commentary: Jon Rollason

Saturday Night and Sunday Morning, 1960, 89 mins, b/w

Production company: Woodfall Film Productions

Executive Producer: Harry Saltzman

Producer: Tony Richardson

Screenplay: Alan Sillitoe, from his novel

Photography: Freddie Francis

Art director: Ted Marshall

Set decoration: Timothy O'Brien

Production manager: Jack Rix

Editing: Seth Holt

Music: Johnny Dankworth and his Orchestra. Special lyrics by David Dearlove

Wardrobe: Sophie Devine, Barbara Gillett

Continuity: Pamela Mann

Sound recording: Peter Handford, Bob Jones

Sound editing: Chris Greenham

Leading players: Albert Finney (Arthur Seaton), Shirley Anne Field (Doreen Gretton), Rachel Roberts (Brenda), Hylda Baker (Aunt Ada), Norman Rossington (Bert), Bryan Pringle (Jack), Robert Cawdron (Robboe), Edna Morris (Mrs Bull), Elsie Wagstaff (Mrs Seaton), Frank Pettitt (Harold Seaton), Avis Bunnage (Blousy woman), Colin Blakely (Loudmouth), Irene Richmond (Mrs Gretton), Louise Dunn (Betty), Peter Madden (Drunken man), Cameron Hall (Mr Bull), Alister Williamson (Policeman), Anne Blake (Civil defence officer)

Night Must Fall, 1964, 101 mins, b/w

Production company: MGM

Executive producer: Laurence P. Bachman

Producers: Albert Finney, Karel Reisz

Screenplay: Clive Exton, adapted from the play by Emlyn Williams

Photography: Freddie Francis

Art director: Lionel Couch

Set decoration: Timothy O’Brien

Production director: Timothy Burrill

Editing: Fergus McDonell, Philip Barnikel

Music/Musical director: Ron Grainer

Sound editing: Malcolm Cooke

Sound mixing: J. B. Smith

Leading players: Albert Finney (Danny), Mona Washbourne (Mrs Bramson), Susan Hampshire (Olivia), Sheila Hancock (Dora), Michael Medwin (Derek), Joe Gladwin (Dodge), Martin Wyldeck (Inspector Willett), John Gill (Foster) 
Morgan: A Suitable Case for Treatment, 1966, 97 mins, b/w

Production company: Quintra Productions, British Lion Films

Producer: Leon Clore

Screenplay: David Mercer, adapted from his teleplay, A Suitable Case for Treatment

Photography: Larry Pizer, Gerry Turpin, gorilla material filmed by Alan Root, Bernard Grzimek

Art director: Philip Harrison

Production manager: Roy Baird

Editing: Tom Priestley

Music: Johnny Dankworth

Costumes: Jocelyn Rickards

Casting: Miriam Brickman

Continuity: Pat Moon

Sound recording: Peter Handford

Dubbing mixer: Hugh Strain

Dubbing editor: John Crome

Leading players: Vanessa Redgrave (Leonie Henderson), David Warner (Morgan Delt), Robert Stephens (Charles Napier), Irene Handl (Mrs Delt), Newton Blick (Mr Henderson), Nan Munro (Mrs Henderson), Bernard Bresslaw (Policeman), Arthur Mullard (Wally), Graham Crowden (Counsel), Peter Cellier (Second counsel), John Rae (Judge), Angus MacKay (Best man), Peter Collingwood (Geoffrey), John Garrie (Tipstaff), Marvis Edwards (Maid), Robert Bridges (Ticket collector)

Isadora, 1968, 153 mins (director's cut), 177 mins (original release print), col.

Production company: Universal

Producers: Robert and Raymond Hakim

Screenplay: Melvyn Bragg, Clive Exton, additional dialogue by Margaret Drabble

Adaptation: Melvyn Bragg

Photography: Larry Pizer (Eastmancolor)

Art directors: Michael Seymour, Miso Senesis, Ralph Brinton

Production designer: Jocelyn Herbert

Set decoration: Bryan Graves, Harry Cordwell, Jocelyn Herbert

Production manager: Eric Rattray

Production manager in Yugoslavia, Italy and France: Henri Baum

Editing: Tom Priestley

Music: Original music composed and conducted by Maurice Jarre; music for the modern dance sequences composed by Anthony Bowles; music for the classic dance sequences arranged and conducted by Anthony Bowles

Choreography: Litz Pisk

Continuity: Ann Skinner 
Sound recording: Ken Ritchie, Maurice Askew

Sound editing: Terry Rawlings

Dialogue editing: Jim Atkinson

Leading players: Vanessa Redgrave (Isadora), John Fraser (Roger Thornton), James Fox (Edward Gordon Craig), Jason Robards (Paris Singer), Ivan Tchenko (Essenin), Vladimir Leskova (Bugatti), Cynthia Harris (Mary Desti), Bessie Love (Mrs Duncan), Tony Vogel (Raymond Duncan), Libby Glenn (Elizabeth Duncan), Ronnie Gilbert (Miss Chase), Wallas Eaton (Archer), Nicholas Pennell (Bedford), John Quentin (Pim), Christian Duvaleix (Armand), David Healy (Sullivan, manager of Chicago theatre), Lucinda Chambers (Deirdre), Simon Lutton Davies (Patrick), Noel Davis (Doctor), Ina de la Haye (Russian teacher), Constantine Yranski (Russian companion), Stefan Gryff (Russian party interpreter), John Brandon (Gospel Billy), Margaret Courtenay, Arthur White, Iza Teller, John Warner, Alan Gifford, Zuleika Robson, Arnold Diamond, Anthony Gardner, Sally Travers, Mark Dignam, Robin Lloyd, Lucy Saroyan, Jan Conrad, Hal Galili, Roy Stephens, Cal McCord, Richard Marner

\section{On the High Road (TV, 1973), 47 mins, col.}

Production company: BBC Television

Producers: Gavin Millar, Melvyn Bragg

Screenplay: English adaptation by Jeremy Brooks, Kitty Hunter Blair, from a one-act play by Anton Chekhov

Photography: Brian Tufano (Eastmancolor)

Design: Tony Abbott

Editing: David Martin

Music arrangement: Anthony Bowles; accordion by Henry Krein

Costumes: Robin Fraser-Paye

Sound: Michael Turner, Stan Morcomb

Leading players: Colin Blakely (Merik), Graham Crowden (Bortsov), David Baker (Fedya), Bob Hoskins (Kuzma), Barry Keegan (Tikhon), Peter Madden (Savva), Vanda Godsell (Maria), Lucy Griffiths (Efimova), Natalie Kent (Nazarovna), Jimmy Gardner (a traveller), David Sterne (Coachman)

\section{The Gambler, 1974, 111 mins, col.}

Production company: Paramount

Producers: Irwin Winkler, Robert Chartoff

Screenplay: James Toback

Photography: Victor J. Kemper (Eastmancolor)

Production designer: Philip Rosenberg

Production manager: Hal Polaire

Editing: Roger Spottiswoode 
Music: Jerry Fielding, based on Symphony No. 1 by Gustav Mahler. 'Una Furtiva Lagrima' sung by Enrico Caruso

Costumes: Albert Wolsky

Casting: Cis Corman

Continuity: Julia Tucker

Sound mixer: Dennis Maitland

Sound editor: Terry Rawlings

Dialogue editor: Derek Holding

Sound re-recordist: Doug Turner

Leading players: James Caan (Axel Freed), Paul Sorvino (Hips), Lauren Hutton (Billie), Morris Carnovsky (A. R. Lowenthal), Jacqueline Brookes (Naomi), Burt Young (Carmine), Carmine Caridi (Jimmy), Carl W. Crudup (Spencer), Vic Tayback (One), Steven Keats (Howie), London Lee (Monkey), Antonio Fargas (Pimp), Stuart Margolin (Cowboy), William Andrews (Basketball coach), Joseph Attles (Singer in park), Ernest Butler (Vernon), Sully Boyar (Uncle Hy), Gregory Rozakis (Joe), Starletta De Paur (Monique), Ed Kovens (Ricky), Lucille Patton (Ricky's wife), Mitch Stein and Jonathan Koshner (College announcers), Charles Polk (Harlem bartender), M. Emmett Walsh (Las Vegas gambler), James Woods (Bank officer), Joel Wolfe (Moe), Allen Rich (Bernie), Ric Mancini (Sal), Raymond Serra (Benny), Baron Wilson (Basketball janitor), Richard Foronji (Donny), Frank Sivero (Donny's driver), Philip Sterling (Sidney), Patricia Fay (Bank teller), Beatrice Winde (Hospital receptionist), Leon Pinkney (Street basketball boy), Alisha Fontaine (Howie's girl), Presley Caton (Monkey's girl), Dick Schaap (Television announcer), Chick Hearn (Radio announcer)

Dog Soldiers (aka Who'll Stop the Rain in the US), 1978, 126 mins, col.

Production company: United Artists

Producers: Herb Jaffe, Gabriel Katzka

Screenplay: Judith Rascoe, Robert Stone, based on the novel Dog Soldiers by Robert Stone

Photography: Richard H. Kline (Technicolor)

Additional photography: Ron Taylor

Production designer: Dale Hennesy. In Mexico, Augustin Ytuarte.

Set designer: Dianne Wager

Set decorator: Robert DeVestel. In Mexico, Enrique Estevez

Production manager: Sheldon Schrager. In Mexico, Alberto Ferrer

Supervising Editor: John Bloom

Assistant film editors: Chris Ridsdale, Mark Conte, Peter Boyle. In Mexico, Carlos Puente Portillo

Special effects: Paul Stewart, Jerry Williams, Kenneth Pepiot, Chuck Dolan Music: Laurence Rosenthal

Source music advisor: Tom Nolan. Songs: 'Philadelphia Fillies' by Del Reeves; 'Put a Little Love in Your Heart' by Jackie DeShannon; 'American 
Pie' by Don McLean; 'I'll Step Down' by Slim Whitman; 'Hey Tonight', 'Who'll Stop the Rain' and 'Proud Mary' by Creedence Clearwater Revival; 'Gimme Some Lovin' by The Spencer Davis Group; 'Golden Rocket' by Hank Snow

Costumes: William Theiss

Casting: Jennifer Shull

Script supervisor: Kathy Thomas. In Mexico, Ana Maria Quintana Production sound mixer: Chris Newman. In Mexico, José B. Carles Re-recording mixer: Bill Rowe

Sound editing: Don Sharpe

Leading players: Nick Nolte (Ray Hicks), Tuesday Weld (Marge Converse), Michael Moriarty (John Converse), Anthony Zerbe (Antheil), Richard Masur (Danskin), Ray Sharkey (Smitty), Gail Strickland (Charmian), Charles Haid (Eddie Peace), David Opatoshu (Bender), James Cranna (Gerald), Timothy Blake (Jody), Joaquin Martinez (Angel), Shelby Balik (Janey), Jean Howell (Edna), Jose Carlos Ruiz (Galindez), John Durren (Alex), Bobby Kosser (Hippie), Wings Hauser (Marine driver), Jonathan Banks (Marine), Michael Bair (Blinded man), Derrel Maury (Soldier), Jan Burrell (Mother), Stuart Wilson (Father), James Gavin (Helicopter pilot), Bill Cross (Radio operator)

The French Lieutenant's Woman, 1981, 123 mins, col.

Production company: United Artists

Producer: Leon Clore

Screenplay: Harold Pinter, from the novel by John Fowles

Photography: Freddie Francis (Technicolor)

Production designer: Assheton Gorton

Art directors: Norman Dorme, Terry Pritchard, Allan Cameron

Set decorator: Ann Mollo

Editing: John Bloom

Assistant editors: Jeremy Hume, Chris Ridsdale

Special effects: Alan Bryce, Nobby Clarke

Music: Carl Davis. Adagio from Mozart's Sonata in D, K 576 , played by John Lill

Costume designer: Tom Rand

Casting director: Patsy Pollock

Continuity: Kay Fenton

Sound recordist: Ivan Sharrock

Sound re-recordist: Bill Rowe

Sound editing: Don Sharpe

Leading players: Meryl Streep (Sarah Woodruff/Anna), Jeremy Irons (Charles Smithson/Mike), Hilton McRae (Sam), Emily Morgan (Mary), Charlotte Mitchell (Mrs Tranter), Lynsey Baxter (Ernestina Freeman), Peter Vaughan (Mr Freeman), Patience Collier (Mrs Poulteney), Leo McKern (Dr Grogan), Colin Jeavons (Vicar), Penelope Wilton (Sonia), Jean Faulds (Cook), Liz Smith (Mrs Fairley), Michael Elwyn (Montague), 
David Warner (Murphy), Gerald Falconetti (Davide), John Barrett (Dairyman), Arabella Weir (Girl on undercliff), Ben Forster (Boy on undercliff), Catherine Willmer (Dr Grogan's housekeeper), Anthony Langdon (Asylum keeper), Edward Duke (Nathaniel), Richard Griffiths (Sir Tom), Graham Fletcher-Cook (Delivery boy), Richard Hope (3rd assistant), Toni Palmer (Mrs Endicott), Cecily Hobbs (Betty Anne), Doreen Mantle (Lady on train), Alun Armstrong (Grimes), Joanna Joseph (Lizzie), Judith Alderson (Red-haired prostitute), Cora Kinnaird (2nd prostitute), Orlando Fraser (Tom Elliott), Fredrike Morton (Girl), Alice Maschler (2nd girl), Matthew Morton, Vicky Ireland, Claire Travers Deacon, Harriet Walter, Janet Rawson, Mia Soteriou, Mary MacLeod, Peter Fraser, Rayner Newmark

Sweet Dreams, 1985, 115 mins, col.

Production company: HBO Pictures, Silver Screen Partners

Producers: Bernard Schwartz, Charles Mulvehill

Screenplay: Robert Getchell

Photography: Robbie Greenberg (Technicolor)

Production designer: Albert Brenner

Art director: David M. Haber

Set design: Frances W. Wells, Kandy Stern

Set decorator: Garrett Lewis

Production manager: Charles Mulvehill

Editing: Malcolm Cooke

Special effects: Burt Dalton, Rodney M. Byrd

Music: Charles Gross

Music consultant: Gregg Perry

Music editor: George Brand

Choreographer: Susan Scanlan

Costumes: Ann Roth

Casting: Ellen Chenoweth

Script supervisor: Wilma Garscadden-Gahret

Sound recording: Jeff Wexler, Don Coufal, James Stuebe

Sound re-recording mixer: Bill Rowe

Sound editors: Derek Holding, Colin Miller

Leading players: Jessica Lange (Patsy Cline), Ed Harris (Charlie Dick), Ann Wedgeworth (Hilda Hensley), David Clennon (Randy Hughes), Gary Basaraba (Woodhouse), John Goodman (Otis), P. J. Soles (Wanda), James Staley (Gerald Cline), Terri Gardner (Girl singer), Courtney Parker (Older Julie), Caitlin Kelch (Sylvia Hensley), Robert L. Dasch (John Hensley), Colton Edwards (Baby Randy), Holly Filler (Madrine), Bruce Kirby (Arthur Godfrey), Jerry Haynes (Owen Bradley), Kenneth White (Big Bill Shawley), Stonewall Jackson (Announcer at Opry), Jake T. Robinson (Biker), Boxcar Willie (Old man in jail), Tony Frank (Bartender), Charlie Walker (Cowboy Copas), Frank Knapp Jr. (Hawkshaw Hawkins), Richard J. Kidney (TV technician), Jack Slater (Recording engineer), Missy 
Proulx (Girlfriend at fair), Aleda Pope (Girlfriend at fair), Carlton Cuse (Sergeant), John E. Davis (Skip Cartmill), John Walter Davis (Stone), Toni Sawyer (Baby nurse), Robert Rothwell (Plastic surgeon), Patricia Allison (Surgery nurse), Patsy's band: William Byrd (bass), John R. Smarr (acoustic guitar), Fred K. Young (drums), Curtis Young (singer/guitar), Michael David Black (singer/guitar)

\section{Everybody Wins, 1990, 98 mins, col.}

Production company: Film Trustees Ltd.

Executive producers: Linda Yellen, Terry Glinwood

Producers: Jeremy Thomas and Ezra Swerdlow

Screenplay: Arthur Miller, adapted from his play, Some Kind of Love Story Photography: Ian Baker (Panavision)

Production designer: Peter Larkin

Art director: Charley Beal

Editing: John Bloom

Music: Mark Isham, additional music by Leon Redbone

Script supervisor: Sandy McLeod

Casting: Ellen Chenoweth

Costume design: Ann Roth

Sound editor: Don Sharp

Sound mixer: Ivan Sharrock

Leading players: Debra Winger (Angela Crispini), Nick Nolte (Tom O'Toole), Will Patton (Jerry), Judith Ivey (Connie), Kathleen Wilhoite (Amy), Jack Warden (Judge Harry Murdoch), Frank Converse (Charley Haggerty), Frank Military (Felix Daniels), Steven Skybell (Father Mancini), Mary Louise Wilson (Jean), Mert Hatfield (Bellanca), Peter Appel (Sonny), Sean Weil (Montana), Timothy D. Wright (Defence Attorney), Elizabeth Ann Klein (Judge), James Parisi (Reporter), R. M. Haley (Driver), T. M. Nelson George (Judge \#2), David Ellis (Stunt co-ordinator), Tim Davison, Don Pulford, Richard Allison (Utility stunts)

Act Without Words I, 2000, 16 mins, col.

Production company: RTE, Channel 4, Bord Scannán na Eireann and Tyrone Productions

Executive producers: Joan Egan, Joe Mulhollan and Rod Stoneman

Producers: Michael Colgan and Alan Moloney

Script: Samuel Beckett

Photography: Seamus Deasy

Art director: Clodagh Conroy

Production designer: Charles Garrad

Editing: Lesley Walker

Music: Michael Nyman

Costumes: Joan Bergin

Script supervisor: Renee Foley-Burke

Leading player: Sean Foley (Man) 


\section{As director, theatre productions}

Gardenia, 1982, by John Guare, with James Woods, JoBeth Williams and Sam Waterston. Manhattan Theater Club, New York.

The Gigli Concert, 1992, by Tom Murphy, with Tony Doyle and Barry Foster. Almeida Theatre, London.

A Doll's House, 1993, by Henrik Ibsen, with Niamh Cusack. Gate Theatre, Dublin.

The Deep Blue Sea, 1993, by Terence Rattigan, with Penelope Wilton and Linus Roache. Almeida Theatre, London. With Penelope Wilton and Colin Firth at the Apollo Theatre, London (Critics' Circle Award and an Olivier nomination). The Apollo production was filmed as a BBC TV play in their 'Performance' series, broadcast 12 November 1994.

Moonlight, 1995, by Harold Pinter, with Blythe Danner and Jason Robards. Roundabout Theatre, New York.

Happy Days, 1996, by Samuel Beckett, with Rosaleen Linehan. Beckett Festival at the Lincoln Center, New York and the Almeida Theatre, London.

A Kind of Alaska, 1998, by Harold Pinter, with Penelope Wilton and Bill Nighy. Donmar Warehouse Theatre, London.

Long Day's Journey into Night, 1998, by Eugene O'Neill, with Donald Moffat and Rosaleen Linehan. Gate Theatre, Dublin.

Ashes to Ashes, 1998-99, by Harold Pinter, with Lindsay Duncan and David Straithairn. Roundabout Theatre/Gramercy Theatre, New York.

A Kind of Alaska, 2001, by Harold Pinter, with Brid Brennan, Stephen Brennan and Penelope Wilton. Pinter Festival at the Lincoln Center, New York.

Landscape, 2001, by Harold Pinter, with Stephen Brennan and Penelope Wilton. Pinter Festival at the Lincoln Center, New York.

The Yalta Game, 2001, by Brian Friel, adapted from Chekhov's 1899 story, The Lady with the Lapdog, with Ciarán Hinds and Kelly Reilly. Dublin Festival. 